\title{
TRUTHMAKERS, ENTAILMENT AND NECESSITY
}

\section{Greg Restall}

Australian Realists are fond of talking about truthmakers. Here are three examples from the recent literature

... suppose $a$ is $F$.. What is needed is something in the world which ensures that $a$ is $F$, some truth-maker or ontological ground for $a$ 's being $F$. What can this be except the state of affairs of $a$ 's being $F$ ? [3, p. 190]

If $\Phi$ entails $\prod$, what makes $\Phi$ true also makes $\prod$ true (at least when $\Phi$ and $\prod$ are contingent). $[8$, p. 32]

The hallowed path from language to universals has been by way of the correspondence theory of truth: the doctrine that whenever something is true, there must be something in the world which makes it true. I will call this the Truthmaker axiom. The desire to find an adequate truthmaker for every truth has been one of the sustaining forces behind traditional theories of universals . . . Correspondence theories of truth breed legions of recalcitrant philosophical problems. For this reason I have sometimes tried to stop believing in the Truthmaker axiom. Yet, I have never really succeeded. Without some such axiom, I find I have no adequate anchor to hold me from drifting onto the shoals of some sort of pragmatism or idealism. And this is altogether uncongenial to me; I am a congenital realist about almost everything, as long as it is compatible with some sort of naturalism or physicalism, loosely construed. [4, pp. 122, 123]

The notion of a truthmaker is a central feature of a number of philosophical programmes. We ought to have a clear understanding of what a truthmaker amounts to, of how it operates, and how it is related to other notions, such as entailment and necessity. There are hints of this in the literature but, as I will show, truthmakers are more problematic than many appear to think.

\section{Dispelling Myths}

John Fox gives an elegant account of truthmakers in his paper 'Truthmaker'. He defines the 'truthmaker axiom' as follows

By the truthmaker axiom I mean the axiom that for every truth there is a truthmaker; by a truthmaker for $A$, I mean something whose very existence entails $A$. [7, p. 189]

As it stands, this axiom needs careful reading. There are a number of ways it could be 
misinterpreted. Firstly, think of a unicorn. Necessarily, if that unicorn exists, then the claim 'a unicorn exists' is true. So, a unicorn is something whose existence would necessitate the claim 'a unicorn exists'. This doesn't mean that the claim 'unicorns exist' is in fact true, because the truthmaker in question, the said unicorn, does not exist. For a truthmaker to be any good at making things true, it needs to exist. ${ }^{1}$

For a second refinement, consider a philosophical view which takes it that all things which exist, exist necessarily - for example, David Lewis' modal realism. For Lewis, existence 'in the broad' is more than being an inhabitant of the actual world. In fact, for Lewis, anything which exists in this broad sense exists necessarily. However, for Lewis, actuality is a contingent matter. What is in the world I am in is a function of the world I am in. And actuality is clearly the appropriate notion for the truthmaker axiom. On this view, a truthmaker for $A$ is some actual object (some inhabitant of the actual world) such that its actuality necessitates $A$.

For another refinement, consider the truth 'there have been at least three performances of Arvo Pärt's Magnificat'. It is hard to see what a truthmaker for this could be other than three performances of Pärt's Magnificat. Is there a single object which comprises these three performances (and whatever else in the world is needed as the truthmaker for the claim)? If there is, it is hopelessly 'gerrymandered'. One performance has taken place in Estonia, one in Berlin, and one in Canberra. The 'object' consisting of these performances is at least a bit strange. Some take this to be an object in its own right. Others do not. So, in deference to those of the second persuasion, let us allow a truthmaker to be an object or objects.

It is also important to realise what the truthmaker axiom is not. It does not posit a unique truthmaker for every claim. (There are many truthmakers for 'someone has swum across the English Channel', for example.) Similarly, the truthmaker axiom does not posit a minimal truthmaker for every claim, where a minimal truthmaker for a claim is a truthmaker which is a part of every truthmaker for that claim. Although people often talk of 'the truthmaker' for a truth, the truthmaker axiom does not postulate any kind of uniqueness. The one truth can be made true in any of a number of different ways.

To sharpen up the truthmaker axiom, we need to take account of entailment. The classical notion of entailment in use ties entailment to necessity. On the classical account, $A$ entails $B$ just when it is impossible for $A \wedge \sim B$ to be true. That is, $A \Rightarrow B$ is $\sim \nabla(A \wedge \sim B)$. This immediately gives rise to our first thesis.

\section{The Classical Entailment Thesis}

For any $A, s$ is a truthmaker of $A$ if and only if $s$ exists, and it is impossible that $s$ exist without $A$. That is, $s=A$ if and only if $E ! s \wedge \sim \diamond(E ! s \wedge \sim A$ ). (Or, for more than one object, $s_{1}, s_{2}, \ldots$ are collectively truthmakers for $A$ if and only if each $s_{i}$ exists, and it is impossible for them each to exist without $A$. In what follows we'll ignore the case where more than one object constitutes the truthmaker, simply for convenience. Nothing of substance hangs on the distinction.)

I am being a little too quick here. Given a particular unicorn, $u$, it may not be the case that in every world in which $u$ exists, it is a unicorn. If this is the case, then $u$, by itself, is not a truthmaker for 'there is a unicorn' in any of the worlds in which it exists. However, the point that a truthmaker must exist for it to be any good as a truthmaker remains unscathed. 
This hypothesis has a number of pleasant consequences for truthmakers and truthmaking. The first is an obvious desideratum of any account of truthmaking, that if $s$ is a truthmaker for $A$, that is, if $s$ makes $A$ true, then $A$ is, in fact, true.

\section{Consequence 1: If $s \vDash A$ then $A$}

The argument is simple. If $s \vDash A$ then $E ! s$, and $\sim \supset(E ! s \wedge \sim A)$ yields $A$ as a simple consequence. $^{2}$

There is also a desirable result connecting truthmaking and conjunction. If something makes both $A$ and $B$ true, then it also makes their conjunction true, and vice versa. We would hope that this would be a consequence of any account of truthmaking.

Consequence 2: $s \vDash A$ and $s \vDash B$ if and only if $s \vDash A \wedge B$

It is also a simple consequence that if something makes $A$ true, then nothing makes $\sim A$ true. As we would hope.

Consequence 3: If $s \vDash A$ then $\sim \exists t(t \vDash A)$

That is enough about the desirable consequences of the strict entailment thesis. It is time to see some of its darker properties. For the first, consider the untoward properties of strict material implication as an account of entailment. It is well known that if $A$ is necessary (so $\sim \sim A$ ) then anything 'entails' $A$. (For any $B, \sim \nabla(B \wedge \sim A)$.) As a result, we have the following consequence.

\section{Consequence 4: If $\square A$ is true, then any existing $s$ is a truthmaker for $A$}

This follows immediately from the definition. Now, this may not be such a problem. There is something quite touching in the view that every particle in the universe (and everything else besides!) is a witness to all necessary truths. ${ }^{3}$ If we read the classical entailment thesis contrapositively $-s$ is a truthmaker for $A$ if and only if were $A$ to fail then $s$ wouldn't exist - then you can at least see why some would be able to swallow the conclusion. After all, were $2+2$ to not equal 4 , then nothing would be quite the same.

However, polemical point scoring about relevance is not my business here. ${ }^{4}$ The problems with the classical entailment thesis are more significant than merely conflicting with our intuitions about what counts as a truthmaker for necessary truth. They threaten collapse of the entire notion of truthmakers. To see this, we need another thesis about truthmakers.

2 Using disjunctive syllogism. Relevantists will baulk at this point. But they have already baulked at the classical entailment thesis.

3 This gives logic a certain grandeur!

- I refer the reader to [1], [2] and [13] in which the relevant polemical points are made more forcefully than I can here. 


\section{The Disjunction Thesis}

For any truthmaker $s, s \vDash A \vee B$ if and only if $s \vDash A$ or $s \vDash B$.

The disjunction thesis seems quite plausible. I will leave discussing the independent merits of the thesis until later. For now, let us see how the disjunction thesis and the classical entailment thesis interact.

\section{Consequence 5: Every truthmaker makes true every truth}

We assume that every instance claim of the form $A \vee \sim A$ is a necessary truth. By one fact we have already seen, every $s$ is a truthmaker for each instance of $A \vee \sim A$. Let $A$ be a truth. So, any $s$ either makes $A$ or $\sim A$ true, by the disjunction hypothesis. Given that $A$ is true, then nothing makes $\sim A$ true. So $s$.is not a truthmaker for $\sim A$. Hence, it must be a truthmaker for $A$.

The result may be called truthmaker monism. We end up with all truthmakers on a par, all making true every truth. This is clearly not acceptable for any philosophically discriminating account of truthmakers.

\section{Jackson and Necessary Truths}

Frank Jackson has noticed at least some of the subtleties associated with truthmakers. His approach to truthmakers (as much as we can discern from the throw-away line in his [8]) denies the classical entailment thesis. We will restate Jackson's claim as follows:

\section{Jackson's Thesis}

If $A$ and $B$ are contingent, and $A$ entails $B$, then if $s \vDash A$, then $s \vDash B$.

So Jackson does not hold that entailment is enough for truthmaking in all cases - in the cases where $A$ is necessary, not everything need amount to a truthmaker for $A$. And that does seem right. Why should my refrigerator count as a truthmaker for the Goldbach conjecture ${ }^{5}$ (or its negation)? We have already seen that assuming the classical account of entailment ( $A$ entails $B$ if and only if it is impossible for $A$ to be true and $B$ to be false) and the entailment condition for truthmakers (if $s \vDash A$ and $A$ entails $B$ then $s \vDash$ $B$ ), is sufficient to show that every truthmaker is a truthmaker for every necessary truth.

Unfortunately, Jackson's revision will not do. The argument is simple. We need only two hypotheses. Firstly, that there is a contingent truth (say $C$ ) and that any truthmaker of a conjunction $A \wedge B$ is a truthmaker for both conjuncts $A$ and $B$. (This is a consequence of the classical entailment thesis, but it is very plausible in its own right.) It is very hard to see how this could fail - if anything makes $A \wedge B$ true, it must surely make $A$ true and make $B$ true as well. Given these two hypotheses, then Jackson's revision still faces problems. Granted even his weaker condition, any truthmaker for a contingent truth still is a truthmaker for every necessary truth. The argument is as follows. Take $C$, a contingent truth; $s$, a truthmaker for $C$; and $A$, a necessary truth. Because $C$ is contingent, so is $C \wedge A$. It is impossible for $C$ to be true while $C \wedge A$ is false (because, by hypothesis, $A$ cannot be false) so on the classical account of entailment, $C$ entails $C \wedge A$. So, by Jackson's condition, $s \vDash C \wedge A$, and hence $s \vDash A$. It

5 The Goldbach conjecture states that every even number is the sum of two primes. 
follows that my refrigerator is still a truthmaker for Goldbach's conjecture (or its negation). Hence, Jackson's thesis is a useless modification. It does no work on its own. When coupled with the classical account of entailment, his proviso of $A$ and $B$ being contingent is irrelevant.

The problem is not restricted to the counter-intuitive nature of everything being a truthmaker for necessary truths. If we grant the disjunction thesis, then even using Jackson's restricted thesis, any truthmaker for a contingent truth is still a truthmaker for every truth. The argument is as before. Since a truthmaker for any contingent claim is a truthmaker for every instance of $A \vee \sim A$, that truthmaker must support either $A$ or $\sim A$. Jackson's thesis does not prevent the collapse into truthmaker monism. ${ }^{6}$

\section{Disjunction: or 'Or and Shmor'}

It is clear that the disjunction thesis is doing a lot of work in these arguments. Without it, we simply have counter-intuitive results. With it, we have a dreadful collapse into monism. Granted the disjunction thesis and either the classical entailment thesis or Jackson's attempted weakening of the thesis, truthmakers cannot draw any distinctions at all other than that between truth and falsity. Perhaps the problem is with the disjunction thesis, and not with the classical entailment thesis.

Consider how the disjunction thesis could fail. Clearly if $s$ makes $A$ true, or it makes $B$ true, then it will make $A \vee B$ true too. So the right-to-left part is trouble-free. The scope for dispute is the step from $s \vDash A \vee B$ to $s \vDash A$ or $s \vDash B$. Both parties in the dispute can agree that if $s \vDash A \vee B$ then there must be something which either makes $A$ true or makes $B$ true. The issue is whether $s$ itself must be such a truthmaker. Suppose it is not. Then by its very existence (which entails $A \vee B$ ) there must be another object, a truthmaker of $A$ or a truthmaker of $B$. As a result, there is a relation of necessitation between distinct objects. The mere existence of $s$ necessitates the existence of some other object. (We must be careful here, for no particular object does it necessitate the existence of that object. Rather, it necessitates the existence of some truthmaker, of either $A$ or of $B$.) But how can a relation of necessitation of this sort hold between objects? One way is for $s$ to be an aggregate of objects, each of which must exist for $s$ to exist. But this kind of relation of necessitation will not do the job we need to fault the disjunction thesis: if one of the parts of $s$ is to be a truthmaker of either $A$ or of $B$, then $s$ will also be a truthmaker of $A$ or of $B$ (as $s$ will necessitate anything any of its essential parts necessitate). So, this kind of necessary connection is of no help for one who wishes to fault the disjunction thesis. Anyone who wishes to fault it must explain the kinds of necessary connections between objects which ground a failure of the thesis. Another (stronger) reason to suppose that disjunction satisfies the disjunction thesis is that if it does not, it would make no difference if it did. Let me explain. Suppose that disjunction does not satisfy the disjunction property. We can define an alternative disjunction (read

An anonymous referee pointed out that my 'trivialising' arguments are reminiscent of Davidson's 'Frege Argument', which is employed, for example, in some essays in his [6]. However, what we do with the results is obviously very different. For Davidson, the failure of intersubstitutability of logically equivalent statements is a sign that a sentential connective is not involved. On my account, on the other hand, we can construct a sentential entailment connective (see section $V$ ) for which substitutability of (classically) equivalent statements fails. 
'shmor') by stipulating that $A \star B$ (' $A$ shmor $B$ ') is made true by a truthmaker if and only if either $A$ or $B$ is made true by that truthmaker. Shmor is just as good a candidate for disjunction as the original variety. Clearly if $A$ is true or if $B$ is true, so is $A \star B$. Because if $A$ is true, then it has a truthmaker, and hence $A \star B$ also has a truthmaker, and so is true. Conversely, if $A \star B$ is true, then one of $A$ and $B$ is true. So, necessarily $A \vee B$ is true if and only if $A \star B$ is true. Or and shmor are necessarily equivalent.

We can also show directly that $A \star \sim A$ must be true. Given that one of $A$ and $\sim A$ is true, one has a truthmaker, and hence, $A \star \sim A$ is true. Since we have shown that $A \star \sim A$ must be true (no matter how the truthmakers decide contingent things), we have assured ourselves that $A \star \sim A$ is necessarily true. Yet, it need not be made true by every truthmaker. Rather, it must be made true by some truthmaker. This is a more straightforward (but obviously less reductionistic) account of the interaction between truthmaking and necessity. The necessary truths are those which must be made true by some truthmaker, no matter how they are 'arranged'.

Given a choice between or and shmor as accounts of disjunction, how do we choose among them? $A \star B$ is necessarily equivalent to $A \vee B$. The disjunction property holds of $A \star B$, by its very construction. Who is to say that $A \star B$ is not the proper way to analyse the disjunction of $A$ and $B$ ? $^{7}$

We have seen that the classical entailment thesis collapses distinctions between truthmakers for necessary truths. We have also seen that the disjunction thesis together with the classical entailment thesis results in monism. Given that simple 'fixes' such as Jackson's do not work to repair the damage, and given that there are independent arguments for the disjunction thesis, we must reject the classical entailment thesis in all its forms as an account of truthmaking.

\section{Truthmakers and Worlds}

After reading an earlier draft of this note, Frank Jackson responded with a number of arguments against the disjunction thesis. He conceded that what I have called 'Jackson's thesis' is an unnecessary amendment to the classical entailment thesis. But he resisted the collapse into monism by rejecting the disjunction thesis. By examining his arguments for rejecting the disjunction thesis, we will be able to see the issues at stake in maintaining a theory of truthmakers.

Jackson's first argument goes as follows. Suppose that $s \vDash A$ for some claim $A$. Then $s \vDash(A \wedge B) \vee(A \wedge \sim B)$ for any $B$ we like. But we can choose $B$ in such a way that neither $s \vDash B$ nor $s \vDash \sim B$. In other words, we deny truthmaker monism. But with the disjunction thesis, $s \vDash A \wedge B$ or $s \vDash A \wedge \sim B$, thus contradicting our supposition that $s \not B$ and $s \not \forall \sim B$.

7 This argument is open to question, at the level of the definition itself. We may ask whether this is a permissible definition. An obvious question is raised by the threat of circularity. We use disjunction in the defining clause, yet we are defining disjunction. This is not a problem, because if you think that $v$ and $\star$ are different, then I can use $v$ in the defining clause. If you take $\star$ and $v$ to coincide, you will note that the definition is circular; but then you have no need of the argument, as you already hold its conclusion. The definition could fail for some other reason - but I cannot at present see what kind of reason this would be. I must leave it for others to show how this definition might fail. 
It ought to be clear that this argument is a way of rephrasing our original argument proving monism from the classical entailment thesis and the disjunction thesis. If we hold to distribution (the equivalence of $s \equiv(A \wedge B) \vee(A \wedge C)$ and $\vDash A \wedge(B \vee C)$, then Jackson's argument relies essentially on the fact that any truthmaker for $A$ is a truthmaker for $A \wedge(B \vee \sim B)$. Or equivalently, any truthmaker makes true every instance of $B \vee \sim B$. So, the argument is simply a modus tollens to our modus ponens. We have shown that the classical entailment thesis leads to trouble, given the disjunction thesis. Jackson's argument shows that, given the classical entailment thesis, the disjunction thesis leads to trouble. This, in itself, is not anything new. What Jackson's argument gives us is another example of where trouble arises. If we grant the disjunction thesis and the principle of distribution, we must deny that any truthmaker for $A$ is also a truthmaker for $A \wedge(B \vee \sim B)$. But that is not a surprise. People have been recommending paring apart $A$ and $A \wedge(B \vee \sim B)$ for many years. ${ }^{8}$

Jackson's second argument relies on what he calls a 'model' of the truthmaker story. For Jackson, we can take a truthmaker for the sentence $A$ to be the set of all worlds in which $A$ is true. Then, $s=A$ just when $A$ is true in all worlds in $s$. A truthmaker is 'actual' just when the actual world is a member of $s$. Given this model, the truthmaker for a disjunction $A \vee B$ is the union of the truthmakers for the disjuncts. So, our argument from distinct existences does not take root - the truthmaker for $A \vee B$ necessitates either the truthmaker for $A$ or the truthmaker for $B$, since together they make up the truthmaker for $A \vee B$, and one of them (at least) must contain the actual world. The truthmakers are not disjoint objects, so that on this picture we have the (somewhat surprising) consequence that a truthmaker for all truths, the singleton set of the actual world, is a part of (a subset of) all actual truthmakers.

This picture certainly provides some kind of counter-example to the disjunction thesis. But is it a satisfying account? There seem to be reasons to think not. Firstly, the truthmakers are what we might call UCLA propositions. They are sets of possible worlds. Some, like David Lewis, take propositions to be UCLA propositions. Whether we agree with this analysis of the nature of propositions or not, it is hard to see what kind of work a UCLA proposition is doing in making a proposition true. This 'model' of the theory of truthmakers is not significantly different to what I will call the simple model.

\section{The Simple Model}

Truthmakers are propositions. A truthmaker is actual if and only if it is true. A truthmaker makes a proposition true just when it entails that proposition.

Given the simple model, the disjunction thesis must fail. $A \vee B$ is a truthmaker for $A \vee B$, but not $A$ nor $B$, for many choices of $A$ and $B$. But the reader is entitled to wonder what has been gained by the simple model of truthmaking, or Jackson's model of truthmaking.

8 For examples and more on this theme see Perry's [9]. Thanks to Gary Malinas for reminding me of this paper, which has themes quite close to the topics at hand here. It will be clear to those familiar with situation semantics that this paper is recasting the ideas of situation semantics for an 'Australian Realist' audience. It should also be mentioned that this approach is somewhat similar to that of van Fraassen's [14]. However, van Fraassen's approach takes facts to be complexes of relations and objects (he follows Russell at least that far). This present project does not follow van Fraassen or Russell down that path. 
A problem with both Jackson's model and the simple model is that they stray too far from the original purpose of the truthmaking account. Neither model takes seriously the view that a truthmaker for $A$ is something which by its very existence ensures that $A$ is true. Truthmakers, on this conception, are parts of the actual world. The way is open, on this approach, to maintain that the only way a piece of the world can ensure that $A \vee B$ is true is for it to ensure that $A$ is true or for it to ensure that $B$ is true. And, furthermore, we can maintain this thesis without rejecting the classical account of entailment wholesale, but rather by enriching our account to pay attention to the fine structure of worlds.

\section{Bigelow and Entailment}

Paying attention to the fine structure of worlds can help us make sense of a passage giving Bigelow's treatment of truthmaking.

Entailment may not be all there is to truthmaking. Not every case of entailment will be a case of truthmaking. But, I claim, every case of truthmaking will be a case of entailment. Perhaps we should formulate truthmaker more delicately, as: 'Whenever something is true, there must be something whose existence entails in an appropriate way that it is true.' This leaves much to be desired, but the main point is that unless the existence of a thing does entail a truth, that thing cannot be an adequate or complete truthmaker for that truth. [4, p. 126]

We have adduced enough cases to show that classical entailment is not all there is to truthmaking. Bigelow's hunch is correct. ${ }^{9}$ But he need not look too far for his account of 'appropriate entailment'. Given what we have seen so far, we can model 'appropriate entailment' alongside its classical cousin.

A world $w\langle W, \subseteq, \models\rangle$ is made up of a collection, $W$, of truthmakers, ordered by inclusion. So, if $s \subseteq s^{\prime}$ then $s$ is a part of $s^{\prime}$. A world comes equipped with a map $=$ from truthmakers to propositions ${ }^{10}$ which satisfies the following.

$s \vDash A \wedge B$ if and only if $s \vDash A$ and $s \vDash B$.

$s \vDash A \vee B$ if and only if $s \vDash A$ or $s \vDash B$.

For every $p$, there is an $s \in W$ where $s \vDash p$ or $s \vDash \sim p$.

For no $s$ does $s \vDash p$ and $s \vDash \sim$ p. ${ }^{11}$

If $s \subseteq s^{\prime}$ and $s \vDash A$ then $s^{\prime} \vDash A$, too. ${ }^{12}$

9 Bigelow also examines the problems of making truthmakers work for negative or universal claims, like 'there are fewer than $n+1$ camels'. He proposes revising the truthmaker thesis to say that for any truth, $A$, either for some collection of objects their existence (appropriately) entails $A$, or $\sim A$ (appropriately) entails the existence of some objects which do not actually exist. This revision is orthogonal to our present purposes.

10 For the moment we consider propositions to be made up inductively from atomic propositions $p_{1}, p_{2}, \ldots$ and their negations, $\sim p_{1}, \sim p_{2}, \ldots$, closing under $\wedge$ and $\vee$. We can then define $\sim(A \wedge B)$ to be $\sim A \vee \sim B$ and $\sim(A \vee B)$ to be $\sim A \wedge \sim B$. This simplifies the treatment of negation in the clauses below.

11 I have written elsewhere $[10,11]$ of the need to question the assumptions of non-contradiction and bivalence. However, the task here is to show how even those who hold staunchly to classical doctrines can understand relevant entailment, through the models of worlds made up of truthmakers.

12 This condition is not essential to the rest of the paper. You can do away with it if you like (or 
We can then say that $A$ is true in $\mathcal{W}$ (written ' $W \models A$ ') just when for some $s \in W, s \vDash A$. It is simple to show that $W \vDash A \vee B$ if and only if $W \vDash A$ or $W \vDash B, W \vDash A \wedge B$ if and only if $w \vDash A$ and $w \vDash B$, and $w \vDash \sim A$ if and only if $w \nvdash A$. So, worlds respect the truth-tabular definitions we learned at our mother's knee. We can then define classical entailment as follows: $A$ classically entails $B$ (written ' $A \rightarrow B$ ') if and only if for every $W$, if $W \models A$ then $W \vDash B$. This definition parallels the classical account of entailment by defining it as truth preservation across all worlds. Similarly, $A$ is a necessary truth just when it is true in all worlds. This notion of necessary truth is also identical to the classical dogma.

However, given the finer structure of worlds, we can define another notion of entailment. $A$ really entails $B$ if and only if, in every world $W$, every truthmaker for $A$ is a truthmaker for $B$. Let us write this as $A \Rightarrow B$. Then it is simple to show that $A \Rightarrow A \vee B$, $A \wedge B \Rightarrow A, A \wedge(B \vee C) \Rightarrow(A \wedge B) \vee(A \wedge C)$ and that if $A \Rightarrow B$ and $B \Rightarrow C$ then $A \Rightarrow C$. However, we do not have $A \Rightarrow(A \wedge B) \vee(A \wedge \sim B)$, nor $A \Rightarrow B \vee \sim B$. Not every truthmaker need make true every necessary truth. Neither do we have that if $A \rightarrow B$, and $s$ $\vDash A$ then $s \vDash B$. For clearly $A \rightarrow(A \wedge B) \vee(A \wedge \sim B)$, but we can find truthmakers for $A$ which need not be truthmakers for $(A \wedge \sim B) \vee(A \wedge \sim B)$.

The entailment $\Rightarrow$ is nearly, but not quite, the first degree entailment of relevant logic (see, for example, [1]). It can be seen that we have $A \wedge \sim A \Rightarrow B$, because there are no truthmakers for $A \wedge \sim A$ in any world. But we can get closer to first-degree entailment by setting $A \Rightarrow_{2} B$ if and only if $A \Rightarrow B$ and $\sim B \Rightarrow \sim A$. Then we do not have $A \wedge \sim A \Rightarrow_{2} B$, but we still have $A \wedge \sim A \Rightarrow_{2} B \vee \sim B .^{13}$

These models are a simple, understandable generalisation of possible worlds semantics. Instead of taking possible worlds as atomic, we look inside possible worlds to see their fine structure of truthmakers. This gives us access to a more discriminating account of entailment, which can support our pre-theoretic notions of truthmaking. I recommend it to all those who seek to understand contemporary work on relevant logic, ${ }^{14}$ and for those who wish to form a robust theory of truthmaking. ${ }^{15}$

Macquarie University

Received May 1995

12 Continued. .

you can trivialise it by saying that truthmakers do not enter into part-of relations with each other, thereby making $s \subseteq s^{\prime}$ only if $s=s^{\prime}$ ). I include it only to show that it can be a part of a semantic picture of trutbmakers.

13 The resulting logic is the first-degree fragment of the relevant logic, $R M$, as a referee pointed out to me.

14 This account of truthmakers only tells us when an entailment is true. It leaves aside the question of what it is that makes an entailment true. This is the point at which the 'ternary relational' semantics for conditionals enters the theory of relevant logics. Whether or not sense can be made of these constructions in terms of truthmakers is a pressing issue. I have addressed some of the issues involved when considering connections between relevant logics and situation semantics. See [12].

15 Thanks to Frank Jackson, Graham Priest, Daniel Nolan, Gary Malinas and Philip Pettit for encouragement, ideas and information. Thanks, too, to three anonymous referees for their helpful comments. 


\section{REFERENCES}

1. Alan Ross Anderson and Nuel D. Belnap, Entailment: The Logic of Relevance and Necessity, vol. 1 (Princeton: Princeton University Press, 1975).

2. Alan Ross Anderson, Nuel D. Belnap, and J. Michael Dunn, Entailment: The Logic of Relevance and Necessity, vol. 2 (Princeton: Princeton University Press, 1992).

3. D.M. Armstrong, 'Classes are States of Affairs', Mind 100 (1991) pp. 189-200.

4. John Bigelow, The Reality of Numbers (Oxford: Oxford University Press, 1988).

5. Donald Davidson, 'Causal Relations', The Journal of Philosophy 64 (1967) pp. 691-703, reprinted in [6].

6. Donald Davidson, Essays on Actions and Events (Oxford: Clarendon Press, 1980).

7. John F. Fox, 'Truthmaker', Australasian Journal of Philosophy 65 (1987) pp. 188-207.

8. Frank Jackson, 'Armchair Metaphysics' in Michaelis Michael and John O'Leary-Hawthorne (eds.), Philosophy in Mind: The Place of Philosophy in the Study of Mind (Dordrecht: Kluwer, 1994).pp. 23-42.

9. John Perry, 'From Worlds to Situations', Journal of Philosophical Logic 15 (1986) pp. 83-107.

10. Greg Restall, 'Deviant Logic and the Paradoxes of Self Reference', Philosophical Studies 70 (1993) pp. 279-303.

11. Greg Restall, 'On Logics Without Contraction', Ph.D. thesis, University of Queensland, January 1994.

12. Greg Restall, 'Information Flow and Relevant Logics' in Jerry Seligman and Dag Westerstahl (eds.), Logic, Language and Computation: The 1994 Moraga Proceedings (Stanford, CA: Center for the Study of Language and Information, 1995) pp. 443-456.

13. Richard Routley, Val Plumwood, Robert K. Meyer, and Ross T. Brady, Relevant Logics and Their Rivals (Atascadero, CA: Ridgeview, 1982).

14. Bas van Fraassen, 'Facts and Tautological Entailments', The Journal of Philosophy 66 (1969) pp. 477-486. 